\title{
Dual-wavelength, ultralong Raman laser with Rayleigh-scattering feedback
}

\author{
A. E. El-Taher, ${ }^{1, *}$ M. Alcon-Camas, ${ }^{1}$ S. A. Babin, ${ }^{2}$ P. Harper, ${ }^{1}$ J. D. Ania-Castañón, ${ }^{3}$ and S. K. Turitsyn ${ }^{1}$ \\ ${ }^{1}$ Photonics Research Group, Aston University, Birmingham, B4 7ET, UK \\ ${ }^{2}$ Institute of Automation and Electrometry, SB RAS, Novosibirsk 630090, Russia \\ ${ }^{3}$ Instituto de Óptica Daza de Valdés, CSIC, Madrid, 28006, Spain \\ *Corresponding author: eltaheae@aston.ac.uk
}

Received December 24, 2009; accepted January 26, 2010;

posted February 26, 2010 (Doc. ID 121869); published March 31, 2010

\begin{abstract}
We present experimental demonstration of a 200-km-long, dual-wavelength Raman laser utilizing two slightly different-wavelength fiber Bragg gratings, one on each side of the fiber span. The obtained results clearly prove the generation of two independent Raman lasers with a distributed "random" Rayleigh scattering mirror forming a cavity together with each of the individual fiber Bragg grating reflectors.

(C) 2010 Optical Society of America

OCIS codes: $190.5650,140.3550,290.5870$
\end{abstract}

Performance of Raman fiber lasers with a gratinglimited fiber cavity has experienced a continuous improvement since their first demonstration in the 1990s [1]. During the past few years, an ultralong Raman fiber laser (URFL) scheme has been implemented as a very attractive light source offering the basis for quasilossless transmission communication links $[2,3]$. URFL features have been studied extensively in this context.

Basically, in an ultralong Raman fiber laser two pump waves propagating in a conventional telecom fiber produce distributed Raman gain for the redshifted (by $\sim 13 \mathrm{THz}$ ) Stokes wave. Forming a cavity by means of fiber Bragg gratings (FBGs) resonantly reflecting forward and backward propagating Stokes waves at the fiber ends results in laser action at the FBG reflection wavelength when the pumpinduced Raman gain exceeds losses in the round trip. For such a conventional symmetric Raman laser scheme, typical laser features such as lasing threshold and nearly linear growth of the Stokes wave power above the threshold with clear longitudinal mode structure with spacing $\Delta=c / 2 n L$ of the generated spectra have been observed at cavity lengths up to $L=84 \mathrm{~km}[4]$.

Recently, lasing in a cavity as long as $270 \mathrm{~km}$ has been reported [5] in which in addition to typical spectra with mode structure new features have been discovered, namely, spectral components generated at wavelengths far from the FBG reflection spectrum with spiky dynamics of laser output, which appears to be more pronounced near the threshold and at nonsymmetric pumping. Moreover, the characteristics of such an asymmetric Raman laser appear almost independent of fiber length in some range, which means that the distributed cavity reflector formed due to the back reflection in the fiber itself, i.e., Rayleigh back scattering (RS) is very significant and strongly influences the characteristics of such laser. A more detailed study of the RS effects in URFLs proved that the distributed feedback due to the Rayleigh back scattering of the light propagating between FBG reflectors may be comparable with the feedback provided by the FBG itself [6]. Conse- quently, Raman lasing in the fiber span limited by a lumped FBG reflector at one side only appears possible due to significant reflection from the RS-based "random" distributed mirror at the other side.

In this Letter, we experimentally demonstrate a 200-km-long, dual-wavelength Raman laser where feedback is based on the combination of two independent lumped FBG reflectors at slightly different wavelengths and distributed Rayleigh scattering in the same fiber span. It will be shown that the two Raman lasers characteristics are independent from each other, and each of them exhibits quite specific temporal behavior and shapes of optical and RF spectra.

The experimental setup for the studied ultralong Raman laser cavity is schematically illustrated in Fig. 1. We used a configuration with pumping at $1455 \mathrm{~nm}$ and Raman lasing at $\sim 1550 \mathrm{~nm}$, for which

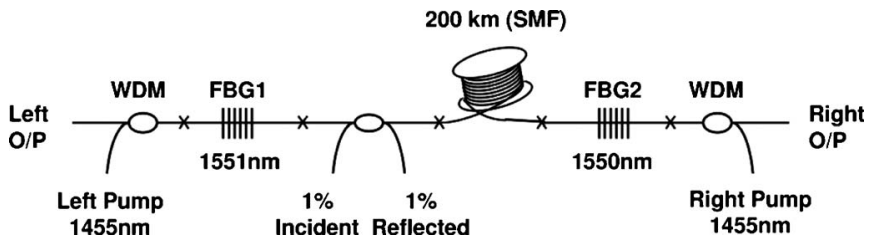

Fig. 1. Schematic depiction of the experimental setup.

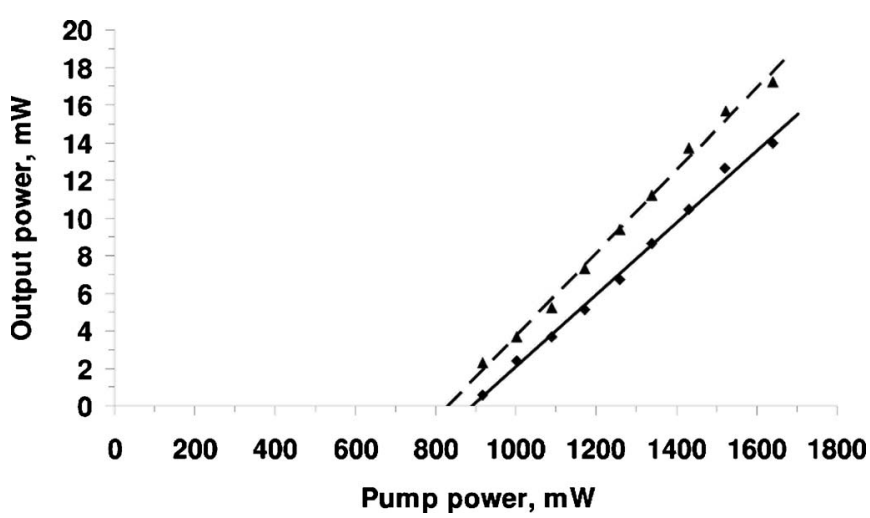

Fig. 2. Laser output power as a function of pump power; squares are the $1550 \mathrm{~nm}$ right laser output power versus right pump power and triangles are the $1551 \mathrm{~nm}$ left laser output power versus left pump power. 

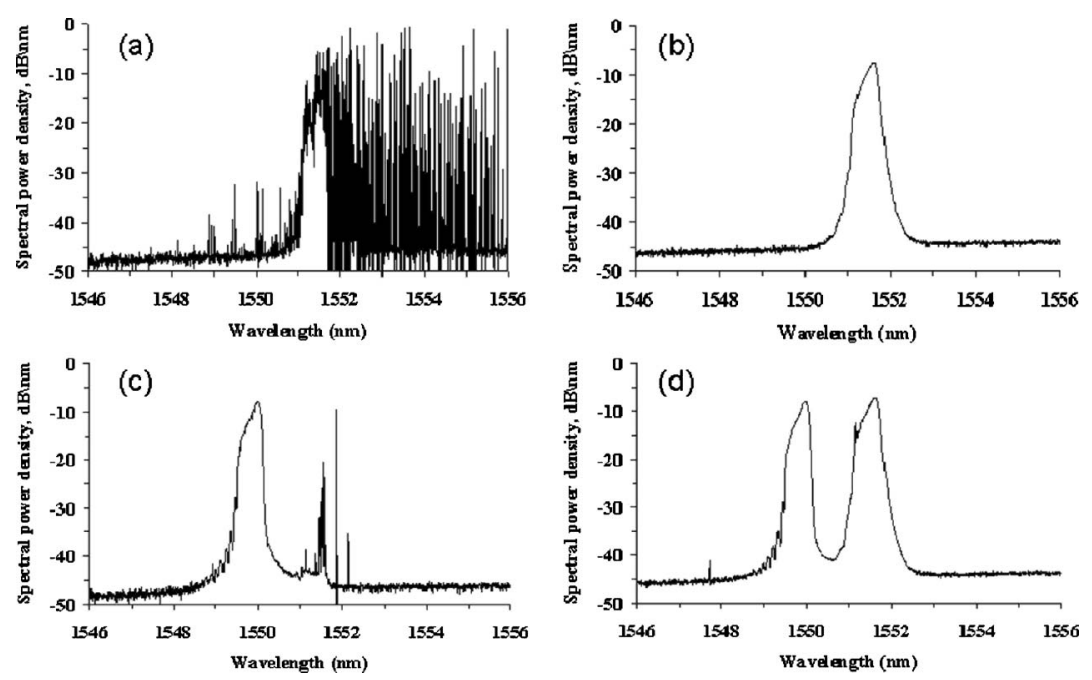

Fig. 3. Optical spectra at various left pump (LP) and right pump (RP) power: (a) $\mathrm{LP}=835 \mathrm{~mW}, \mathrm{RP}=0 \mathrm{~mW}$; (b) $\mathrm{LP}=965 \mathrm{~mW}, \mathrm{RP}=0 \mathrm{~mW}$; (c) $\mathrm{LP}=735 \mathrm{~mW}, \mathrm{RP}=1175 \mathrm{~mW}$; (d) $\mathrm{LP}=965 \mathrm{~mW}, \mathrm{RP}=1175 \mathrm{~mW}$.

losses are minimal. This offers the possibility of lengthening the URFL cavity, by analogy with the schemes in $[5,6]$. Two pump lasers were coupled into the fiber span through wavelength division multiplexers (WDM). Two highly reflective FBGs with reflection coefficient $R \sim 98 \%$ and bandwidth of $\sim 0.4 \mathrm{~nm}$ at different central wavelengths are spliced to the fiber span at the points marked by crosses on Fig. 1. FBG1 with a central Bragg wavelength of $1551 \mathrm{~nm}$ is spliced to the left end of the span and FBG2 with a central Bragg wavelength of $1550 \mathrm{~nm}$ is spliced to the right end of the span. The width of the reflection profile of the FBGs is less than the $1 \mathrm{~nm}$ separation between their Bragg wavelengths. Fiber ends were angle cleaved to eliminate reflection.

In this experiment, a 200-km-long spliced sectioned SMF fiber span is used. To measure the Raman laser characteristics of the waves propagating in opposite directions inside the cavity, a splitter with two $1 \%$ ports was inserted near the left FBG. Optical spectra were measured with a high resolution (0.01 $\mathrm{nm}$ ) optical spectrum analyzer (OSA). The RF spectra characterizing laser intensity fluctuations were also measured using photodiode and electric spectrum analyzer (ESA) with a resolution of $\sim 100 \mathrm{~Hz}$, and the corresponding time domain intensity behavior was analyzed by means of an oscilloscope with a $50 \mathrm{ps}$ temporal resolution.

In spite of the negligibly small overlapping of the FBGs reflection spectra, the scheme pumped by $\sim 1 \mathrm{~W}$ power at $1455 \mathrm{~nm}$ from each side exhibits clear laser properties at both FBG wavelengths, but the output appears to be directional: at the left output end, $1551 \mathrm{~nm}$ radiation (corresponding to the reflection maximum of the left FBG1) is dominant, whereas at the right end $1550 \mathrm{~nm}$ radiation (FBG2) provides the main contribution. Besides, the left output power at $1551 \mathrm{~nm}$ depends only on the injected left pump power (right pump power may be even zero), whereas the right output power at $1550 \mathrm{~nm}$ depends on the right pump power only, without any significant cross correlation. Power curves for each wavelength are shown in Fig. 2 versus the corre- sponding pump power; they are almost linear and close to each other. The slight difference in thresholds and slopes may be explained by different losses of FBGs, WDM couplers and splices at the left and the right parts of the scheme. Thus, it can be reasoned that for such a long cavity, most radiation coming from the left-side grating is reflected via Rayleigh backscattering before even reaching the right side grating, and vice versa. Hence, two independent cavities are created within the same fiber span. Moreover, since most of the Raman gain takes place close to each of the fiber ends, the two cavities can be considered to be independently pumped too, as long as we are near the threshold.

In Fig. 3, optical spectra measured inside the cavity via the $1 \%$ incident port are shown for different pump powers. With a single pump (e.g., for the left pump) the spectra behave similarly to those in a single FBG laser [6]: near the threshold (that corresponds to the left pump power of $\sim 730 \mathrm{~mW}$ ) the optical spectrum consists of a narrow peak corresponding to the left FBG reflection spectrum centered at $1551 \mathrm{~nm}$ and broadband spiky continuum; see Fig. $3(\mathrm{a})$. At the same time, the measured RF spectrum shows no sign of mode beating (modes corresponding to a cavity round-trip frequency $f=c / 2 \mathrm{Ln}$ would be expectable for a closed grating cavity, as seen in [5]). Instead, a single RF peak at $\sim 11 \mathrm{GHz}$ is clearly seen (corresponding to the Brillouin scattering Stokes shift), see Fig. 4(a), similar to that on a self-Q-switched Raman laser with RS-Brillouin mirror [7], see also [8]. In the time domain, see Fig. 5(a),
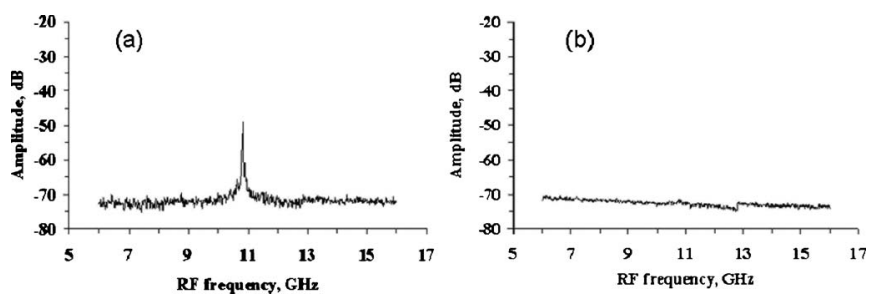

Fig. 4. $\quad \mathrm{RF}$ spectra at (a) $\mathrm{LP}=835 \mathrm{~mW}, \mathrm{RP}=0 \mathrm{~mW}$; (b) $\mathrm{LP}=965 \mathrm{~mW}, \mathrm{RP}=0 \mathrm{~mW}$. 

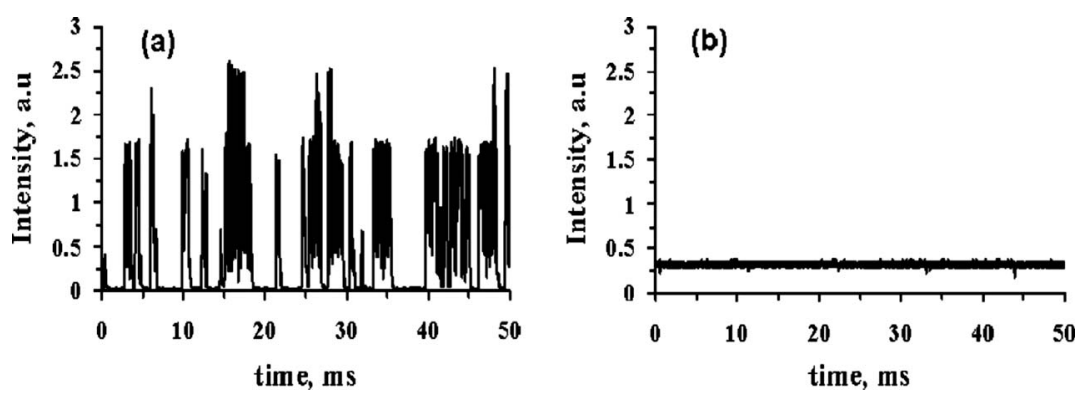

Fig. 5. Time-domain behavior at (a) $\mathrm{LP}=835 \mathrm{~mW}, \mathrm{RP}=0 \mathrm{~mW}$; (b) $\mathrm{LP}=1060 \mathrm{~mW}, \mathrm{RP}=0 \mathrm{~mW}$.

stochastic pulses are generated in this regime. Well above the threshold, only the peak corresponding to the FBG reflection spectrum is present, see Fig. 3(b), while the Brillouin peak in RF spectrum disappears and the operation of the Raman laser remains quasistationary, see Figs. 4(b) and 5(b). For the right pump only, the pictures are similar but the generation occurs at the right FBG reflection wavelength. Well above threshold, a single $1550 \mathrm{~nm}$ peak is observed, while near the threshold the spectrum contains noise in addition to the FBG peak, while temporal behavior is spiky with an $11 \mathrm{GHz}$ peak in the $\mathrm{RF}$ spectrum, similar to the case of the single left pump. With the addition of the second pump an additional peak at the second wavelength appears which is noisy at the threshold [Fig. 3(c)].

With the two pumps well above the threshold, two separated peaks are present in the optical spectra corresponding to different FBGs reflection at 1550 and $1551 \mathrm{~nm}$, see Fig. 3(d), while the RF spectrum shows no $11 \mathrm{GHz}$ peak and intensity is quasistationary in the time domain, similarly to Figs. 4(b) and 5(b). So, two laser cavities formed by each FBG and RS distributed feedback in the same fiber span operate independently, each of which is similar to the single FBG RS-supported Raman laser described in [6]. Thus, the obtained results clearly confirm that a distributed Rayleigh scattering "random" mirror can form a cavity together with each FBG at pumping from this FBG side of the span providing stationary lasing well above threshold. When the distributed Raman gain exceeds the losses in such a distributed cavity, stable laser operation appears possible at the wavelength of corresponding FBG. This laser is "modeless" but its optical spectrum is limited by the FBG reflection curve. When two pumps are applied simultaneously, lasing is possible at two simultaneous wavelengths corresponding to those of both FBGs. These two lasers based on two FBG-RS cavities in a single span are shown to operate independently and stably. Note that this regime does not involve Brillouin scattering leading to self-Q-switched pulsed operation $[7,8]$. Besides, the combined Rayleigh-Brillouin feedback is also utilized for generation of Brillouin-shifted multiple-wavelength combs [9].

In conclusion, we present an experimental demonstration of a 200-km-long, dual-wavelength CW Raman laser based on the Rayleigh scattering utilizing two different fiber Bragg gratings at both sides of the same fiber span. The obtained results clearly prove that the two Raman lasers are stable and independent from each other, since a Rayleigh scattering distributed "random" mirror forms a cavity together with a single FBG independent on the other reflector. In this sense, the studied configuration has no principle length limit, in contrast to the cavity formed by equal FBGs in which a standing wave between FBGs is not formed above a critical cavity length of $\sim 270 \mathrm{~km}$ [5] while RS-based operation is also possible above the limit.

On the basis of the obtained results, multiwavelength ultralong fiber lasers with random distributed feedback and multiple gratings at both ends may be developed with the potential for multiwavelength secondary pump providing extended bandwidth of quasilossless transmission. The reported new laser scheme has a great potential for applications in longdistance communications, as well as in distributed sensor systems based on lasing schemes involving the Rayleigh scattering and reflection from FBGs with different wavelengths [10].

\section{References}

1. S. G. Grubb, T. Strasser, W. Y. Cheung, W. A. Reed, V. Mizrahi, T. Erdogan, P. J. Lemaire, A. M. Vengsarkar, and D. J. Digiovanni, in Optical Amplifiers and Their Applications, Proceedings (Optical Society of America, 1995), pp. 197-199.

2. J. D. Ania-Castañón, T. J. Ellingham, R. Ibbotson, X. Chen, L. Zhang, and S. K. Turitsyn, Phys. Rev. Lett. 96, 023902 (2006).

3. J. D. Ania-Castañón, V. Karalekas, P. Harper, and S. K. Turitsyn, Phys. Rev. Lett. 101, 123903 (2008).

4. S. A. Babin, V. Karalekas, P. Harper, E. V. Podivilov, V. K. Mezentsev, J. D. Ania-Castañón, and S. K. Turitsyn, Opt. Lett. 32, 1135 (2007).

5. S. K. Turitsyn, J. D. Ania-Castañón, S. A. Babin, V. Karalekas, P. Harper, D. Churkin, S. I. Kablukov, A. E. El-Taher, E. V. Podivilov, and V. K. Mezentsev, Phys. Rev. Lett. 103, 133901 (2009).

6. A. Babin, A. El-Taher, P. Harper, D. V. Churkin, S. I. Kablukov, E. V. Podivilov, and S. K. Turitsyn, Conference on Lasers and Electro-Optics-Europe I International Quantum Electronics Conference (IEEE, 2009), Paper CJ5.6.

7. G. Ravet, A. A. Fotiadi, M. Blondel, and P. Megret, Electron. Lett. 40, 528 (2004).

8. S. V. Chernikov, Y. Zhu, J. R. Taylor, and V. P. Gapontsev, Opt. Lett. 22, 298 (1997).

9. A. K. Zamzuri, M. I. M. Ali, A. Ahmad, and R. Mohamad, Opt. Lett. 31, 918 (2006).

10. O. Frazao, C. Correia, J. L. Santos, and J. M. Baptista, Meas. Sci. Technol. 20, 045203 (2009). 